Check for updates

Cite this: RSC Adv., 2018, 8, 38445

Received 28th September 2018 Accepted 7th November 2018

DOI: $10.1039 / c 8 r a 08063 a$

rsc.li/rsc-advances

\section{A variable-temperature X-ray diffraction and theoretical study of conformational polymorphism in a complex organic molecule (DTC) $\dagger$}

\author{
Andrea Gionda, ${ }^{a}$ Giovanni Macetti, ${ }^{a b}$ Laura Loconte, ${ }^{a}$ Silvia Rizzato, (D) ${ }^{a}$ \\ Ahmed M. Orlando, ${ }^{a}$ Carlo Gatti id bc and Leonardo Lo Presti (iD *abc
}

\begin{abstract}
Two conformational crystal polymorphs of 3-diethylamino-4-(4-methoxyphenyl)-1,1-dioxo- $4 H-1 \lambda^{6}, 2-$ thiazete-4-carbonitrile (DTC) have been analyzed in the $100 \mathrm{~K}$-room temperature range by single crystal $\mathrm{X}$-ray diffraction and high quality DFT calculations. DTC has strongly polar as well as aliphatic substituents but no hydrogen bonding groups, and thus qualifies as a test molecule for the relative importance of electrostatic vs. dispersion-repulsion terms. The two polymorphs have the same $P 2_{1} / n$ space group and differ by a flipping of the $-\mathrm{OCH}_{3}$ group, the two conformations being almost equienergetic and separated by a low barrier. The system is monotropic in the observed temperature range with nearly identical thermal expansion coefficients and energy-temperature slopes, one phase consistently predicted to be more stable in agreement with the relative ease of appearance. Energy decompositions show that the electrostatic term is dominant and stabilizes with decreasing temperature. Dispersion and repulsion show the expected behavior, the former becoming more stabilizing at lower temperature in contrast with increasing repulsion at higher density. Absolute values and trends are very similar in the two phases, explaining the small total energy difference. Geometrical analyses of intermolecular contacts using fingerprint plots, as well as the study of molecular dipole moments as a function of $T$ in the framework of the Quantum Theory of Atoms in Molecules, reveal more details of phase stability.
\end{abstract}

\section{Introduction}

Different polymorphs have different intensive physical properties. Prediction and control of the most probable solid-state forms would be highly desirable in several cutting-edge technological areas, such as the development of functional materials ${ }^{1}$ and drug research. ${ }^{2}$ Unfortunately, polymorphism is far from being clearly understood. ${ }^{3}$ In a well-known commentary dating back more than a decade ago, ${ }^{4}$ Desiraju admitted that

${ }^{a}$ Università degli Studi di Milano, Department of Chemistry, Via Golgi 19 I-20133 Milano, Italy.E-mail: leonardo.lopresti@unimi.it

${ }^{b}$ Centre for Materials Crystallography, Århus University, Langelandsgade 140, DK8000 Århus C, Denmark

'Istituto di Scienze e Tecnologie Molecolari, Italian CNR, Via Golgi 19 I-20133 Milano, Italy

$\dagger$ Electronic supplementary information (ESI) available: Details of the energy decomposition schemes explored in this work; crystallographic and refinement details; eigenvalues of the translations and libration tensors describing the rigid body motion of the DTC asymmetric unit; TLS $+\mathrm{H}$ corrected fractional coordinates; conformational descriptors; crystal packing; hydrogen bonded contacts at $100 \mathrm{~K}$ and RT; optimized lattice parameter of real and virtual polymorphs; total cohesive energy per molecule (M06 and PBE0+D); molecule-molecule interaction energies. CCDC 1821383-1821396. For ESI and crystallographic data in CIF or other electronic format see DOI: $10.1039 / \mathrm{c} 8 \mathrm{ra} 08063 \mathrm{a}$ "the question of polymorphism [...] takes the difficult problem of" crystal structure prediction (CSP) "to an even higher level". Crystal nucleation and growth kinetics are as important as thermodynamics in governing the crystallization process, ${ }^{5,6}$ and there is no way to know in advance whether the Ostwald poly$\operatorname{morph}(\mathrm{s})$ - the first appearing one(s) - will be also thermodynamically favoured or not. ${ }^{4,7}$ Accordingly, it cannot be predicted if a change in the crystallization conditions will result in different crystal structures., ${ }^{8,9}$ In the last decade, several computational recipes ${ }^{10}$ have been proposed to face the CSP problem; sometimes, impressive successes have been achieved, ${ }^{10,11}$ but a predictive theory of crystal structure is still lacking. ${ }^{12,13}$ On the other hand, the recent flourishing of accurate tools for exploring in depth crystal energy landscapes ${ }^{10,14,15}$ led to the paradox that the simulations often suggest more thermodynamically stable structures than known polymorphs. ${ }^{6}$ In molecular crystals, lattice cohesive energies are various orders of magnitude lower than bond dissociation energies ${ }^{15}$ and rely, besides the electrostatic, on non-covalent interactions (NCI), often competing with one another. ${ }^{16-18}$ What likely drives the system toward a minority of stable structures, preventing at the same time frequent abrupt transformations from one polymorph to another, are the high energy barriers ${ }^{6,19}$ that keep close minima far apart, in conjunction with the early selection 


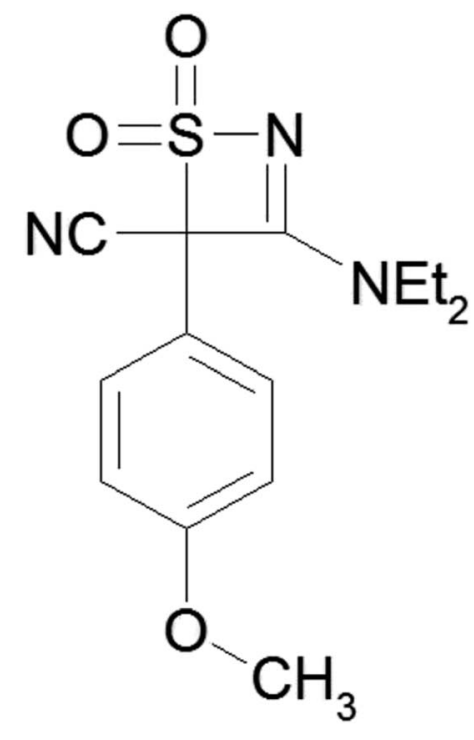

Scheme 1 Molecular structure of DTC.

of (meta)stable clusters at the initial stages of the nucleation event. ${ }^{20}$ This poses into question the general reliability of brute force CSP methods, which are based on the comparison of computer-generated stable structures at $0 \mathrm{~K}$. A better understanding of finite- $T$ effects could come from the knowledge of how the electrostatic interactions and the relative weights of competing NCI networks evolve as a function of macroscopic thermodynamic variables. Such informations are, however, rather scanty.

In this work, a multi-temperature study of the two known conformational polymorphs ${ }^{21-23}$ of 3-diethylamino-4-(4-methoxyphenyl)-1,1-dioxo- $4 H-1 \lambda^{6}, \quad$ 2-thiazete-4-carbonitrile (DTC, Scheme $1, \mathrm{C}_{14} \mathrm{H}_{17} \mathrm{~N}_{3} \mathrm{O}_{3} \mathrm{~S}$ ) is reported. The main objectives are (i) to study correlations between molecular conformation and interaction energetics in the two forms, providing a rationale for their relative stability, and (ii) to investigate how and why electrostatic interactions and the various NCI patterns depend on temperature. The title compound is strongly polar, ${ }^{23}$ lacks $-\mathrm{OH}$ and $-\mathrm{NH}$ hydrogen bond (HB) donor groups and does not undergo structural phase transitions in the $T$ range here explored. Thus, it represents an ideal test case to single out the mutual interplay of dispersive and electrostatic interactions as possible structure-determining factors.

\section{Methods}

\subsection{Crystallization}

The structure of the form A was originally solved in 2003, after crystallizing freshly synthesized DTC by slow evaporation from a $1: 1 \mathrm{CH}_{2} \mathrm{Cl}_{2}: \mathrm{Et}_{2} \mathrm{O}$ mixture. ${ }^{21,22}$ In 2010 , the form $\mathrm{B}$ was discovered upon dissolution of the aged compound in warm distilled water and subsequent recrystallization by slow evaporation at $\mathrm{RT}^{22}$ The scarceness of the synthesized material ${ }^{21}$ prevented further crystallization screenings. In the present study, the same specimen of the B phase employed in a previous work ${ }^{22}$ was used.

\subsection{X-ray diffraction}

Diffraction experiments were performed on a three-circle Bruker SMART APEX II diffractometer equipped with a CCD area detector and an Oxford Cryostream $\mathrm{N}_{2}$ gas blower. A series of, on average, $99.9 \%$ (form A) and $100.0 \%$ (form B) complete spheres of diffraction data were recorded up to a maximum average resolution of $0.768 \AA$ (form A) and $0.769 \AA$ (form B). $\omega$ scans ( $0.5 \mathrm{deg}$ per frame) with graphite-monochromated Mo K $\alpha$ radiation were performed at 7 temperatures among $100 \mathrm{~K}$ and RT. The SAINT+ software package ${ }^{24}$ was used for data reduction, whereas corrections for beam anisotropy and specimen absorption were carried out with SADABS. ${ }^{25}$ All the structural least-squares refinements were performed by shelxl, ${ }^{26}$ starting from the previously determined phases at room temperature. ${ }^{21,22}$ Tables S1 and S2 in the ESI $\uparrow$ show the main specs of data collections and refinements.

\subsection{Rigid body analysis and hydrogen atoms treatment}

The experimental atomic coordinates were corrected for the rigid-body libration of the asymmetric unit, ${ }^{27}$ as computed through the Schomaker and Trueblood TLS decomposition procedure $^{28}$ of the experimental Debye-Waller tensors. The program THMA14 $\mathrm{c}^{29}$ was employed to this scope. Then, all the $\mathrm{C}-\mathrm{H}$ bond distances were renormalized a posteriori to neutronderived estimates ${ }^{30}$ by Mercury v3.931 ("H-correction"). ${ }^{31}$ It must be stressed that this procedure ensures a high quality of the experimental geometries, as correction for rigid-body motion avoids the typical underestimation of covalent bond lengths, which especially affects substituents far from the inertial axes. ${ }^{27}$ Actually, the lacking of accurate experimental models is often a bottleneck against computational methods aimed at the correct energy ranking of polymorphs. ${ }^{32}$ As expected, the higher the temperature, the higher is the TLS correction (Fig. S3 ESI $\dagger$ ). Tables S3 and S4 ESI $\dagger$ report on the eigenvalues of the TLS tensors for both crystal forms and Tables $\mathrm{S} 5$ and S6 $\dagger$ ESI provide (TLS $+\mathrm{H}$ )-corrected atomic coordinates in $f(T)$.

\subsection{Solid-state quantum simulations}

Following commonly accepted procedures, ${ }^{33-36}$ single-point periodic DFT calculations were carried out on the accurate Xray structures of DTC polymorphs (Section 2.3) with the CRYSTAL14 program. ${ }^{37}$ The Minnesota-class meta-GGA M06 functional ${ }^{38}$ was used throughout, in conjunction with the Peintinger's pob-TZVP basis set ${ }^{39}$ specifically developed for solid state studies. M06 includes non-local terms that depend on the kinetic energy density. Therefore, it can partially retrieve correlation effects related to dispersion interactions and it is largely employed to this purpose..$^{40-42}$ An attempt to include dispersion terms semiempirically, with a PBE0+D functional, led here to positive cohesive energies (see Section 3.6 below). No dipolar approximations were exploited for bielectronic integrals, while truncation criteria of $10^{-8}$ and $10^{-16}$ were used (TOLINTEG $8 \quad 8 \quad 8 \quad 8 \quad 16$ ). ${ }^{37}$ A level shifter of $0.3 \mathrm{~h}$ and a $30 \%$ mixing of the Kohn-Sham matrix coefficients were applied to 
subsequent SCF cycles to accelerate convergence. ${ }^{37}$ A $4 \times 4 \times 4$ sampling of the independent part of the first Brillouin zone defined the $k$-space grid for exploiting the SCF procedure. The Atoms in Molecules (AIM) topological analysis ${ }^{\mathbf{4 3}}$ of the wavefunction-derived primary charge density was carried out by the TOPOND14 program $^{44}$ embedded in the main CRYSTAL14 code. $^{37}$ A lower theory level (M06/86-311G**(sulphur) ${ }^{45}+6$ $31 \mathrm{G}^{*}$ (other atoms) ${ }^{46}$ ) was employed to fully optimize the A and $\mathrm{B}$ lattices at $T=0 \mathrm{~K}$. The Hirshfeld surface analysis ${ }^{47}$ was performed with the CrystalExplorer17 (ref. 48) suite.

\subsection{In vacuo quantum simulations}

The Gaussian09 program ${ }^{49}$ was employed throughout. Isolated molecular pairs were computed at their in-crystal geometries using the same M06/pob-TZVP theory level as described above (Section 2.4). The energy barrier for the interconversion between the A and B conformers in the two crystal forms was estimated by a transition-state optimization in the gas phase through the Synchronous Transit-Guided Quasi-Newton (STQN) method. ${ }^{50}$

\subsection{Cohesive energies}

M06 crystal cohesive energies per molecule, $E_{\text {coh }}$, are defined as: ${ }^{51,52}$

$$
E_{\mathrm{coh}}=E_{\mathrm{bulk}} / Z-\left(E_{\mathrm{iso}}+\Delta E_{\mathrm{rel}}-\Delta E_{\mathrm{BSSE}}\right)
$$

$E_{\text {bulk }}$ is the total electronic energy per cell at the TLS $+\mathrm{H}$ corrected (Section 2.3) experimental geometry; $Z$ is the number of formulae in cell ( 4 for DTC); $E_{\text {iso }}$ is the energy of the isolated DTC molecule frozen at its solid-state conformation. The negative $\Delta E_{\text {rel }}$ corrective term accounts for the relaxation energy, i.e. for the energy difference between the fully relaxed molecule in vacuo and the same molecule at its solid-state geometry. Eventually, $\Delta E_{\mathrm{BSSE}}$ is the positive correction for basis set superposition error in the solid state according to Boys and Bernardi. ${ }^{37,53}$ Being based on single-point geometries, this model lacks any vibrational zero-point correction. The accurate study of lattice vibrations, as well as their effects on the Gibbs free energies, goes beyond the purpose of the present work. In any case, the very similar behavior of the cell volume of the A and $\mathrm{B}$ forms as a function of $T$, as well as the identical thermal expansion coefficients (see infra), likely indicate that vibrational effects are very similar in both polymorphs.

\subsection{Energy decomposition}

It is known that cohesive $\left(E_{\text {coh }}\right)$ and molecule-molecule interaction energies $\left(E_{\text {int }}\right)$ can be decomposed into Coulomb-polarization $\left(E_{\mathrm{c}}, E_{\mathrm{p}}\right)$ and dispersive-repulsive $\left(E_{\mathrm{dis}}, E_{\mathrm{rep}}\right)$ terms once a suitable energy partitioning scheme is available:

$$
E_{\mathrm{tot}}=E_{\mathrm{c}}+E_{\mathrm{p}}+E_{\mathrm{dis}}+E_{\mathrm{rep}}
$$

with $E_{\text {tot }}$ being either $E_{\text {coh }}$ or $E_{\text {int }}$. As all the partition schemes are necessarily arbitrary (see Sections 3.7 and S1, S4 ESI $\dagger$ ), we applied various recipes to the structures of A and B polymorphs in the $100 \mathrm{~K}$ - RT $T$ range, to look for model-independent trends of $T$-driven changes. The interested reader can found full methodological details and the corresponding results in Sections S1 and S4 of the ESI. $\dagger$ Here (Section 3.7), the outcomes of Spackman's Electron Charge Density Approach (ECDA), ${ }^{54,55}$ based on the Buckingham's tensor formulation ${ }^{56}$ and implemented in PAMoC, ${ }^{57}$ were discussed.

\subsection{Quality assessment and reproducibility}

CCDC 1821383-1821396 contain the full supplementary crystallographic data for this work. PLATON ${ }^{58}$ checkcif files were also deposited as part of the ESI. $\dagger$ All the quantum simulations were performed using commercial programs, whereas PAMoC is available free of charge for Academic institutions from the following websites: https:/www.pamoc.it; http:// www.angelogavezzotti.it; http://crystalexplorer.scb.uwa.edu.au/.

\section{Results and discussion}

\subsection{Molecular conformation}

The title compound is chiral and its known A and B polymorphs are both centrosymmetric racemates. Fig. 1 shows the geometry of DTC at 100(2) K, together with the atom-numbering scheme. The most striking difference between A and B consists in the relative orientation of the terminal $-\mathrm{OCH}_{3}$ group. At $T=100 \mathrm{~K}$, the phenyl-OCH $\mathrm{OH}_{3}$ torsion angle, $\tau(\mathrm{C} 1-\mathrm{O} 1-\mathrm{C} 2-\mathrm{C} 5)$, is $-7.1(2) \mathrm{deg}$ for the A polymorph and -174.4(1) deg for the B one (Fig. 1, inset). Other conformational differences are significantly smaller. They concern a small rotation of the phenyl group with respect to the thiazete 4-membered ring around the $\mathrm{C} 7-\mathrm{C} 8$ bond and a minor adjustment of the terminal ethyl chains, C11-C12 and C13-C14 (Fig. S4 ESI $\dagger$ ). In form A, the thiazete ring is invariably almost completely planar, with a weighted average of absolute torsion angles well below $5 \mathrm{deg}$; in the form B, a very small puckering was detected only at low $T$, with an amplitude coefficient $^{59} Q(2)$ never greater than $-0.07 \AA$ for $T \leq 140 \mathrm{~K}$.

\section{$3.2 \quad T$-dependent phenomena}

The two crystal forms share the same centrosymmetric space group $P 2{ }_{1} / n$ and have very similar cell volumes (Fig. S5 $\operatorname{ESI} \dagger$ ), differing at most by $0.9 \%$ at RT and by $0.5 \%$ at $100 \mathrm{~K}$. Also densities (at $100 \mathrm{~K}, \rho(\mathrm{A})=1.389 \mathrm{~g} \mathrm{~cm}^{-3} ; \rho(\mathrm{B})=1.383 \mathrm{~g} \mathrm{~cm}^{-3}$ ) and mean coefficients of thermal expansion in the $100 \mathrm{~K}-\mathrm{RT}$ range $\left(\alpha(\mathrm{A})=1.7(3) \times 10^{-4} \mathrm{~K}^{-1} ; \alpha(\mathrm{B})=1.9(2) \times 10^{-4} \mathrm{~K}^{-1}\right.$, computed on the change of the cell volume) are almost identical as well. A similar behaviour was reported also for other organic crystals, ${ }^{60-62}$ though not being a general rule. ${ }^{63-65}$ No phase transitions were identified in either sample (see Tables S1 and S2 ESI $\dagger$ ). In both phases, the DTC conformation does not significantly depend on $T$ (Fig. S4 ESI $\dagger$ ). The molecular units also maintain a constant relative orientation with respect to reference system. Comparing the inertial eigenvectors among $100 \mathrm{~K}$ and RT, it turns out that the asymmetric unit undergoes a $0.41 \mathrm{deg}$ wide rotation in form A and $0.36 \mathrm{deg}$ in form B. The $3-4 \%$ large reduction of cell volume (Fig. S5 ESI $\dagger$ ) forces the molecules to come closer and closer. On average, the centre-of- 


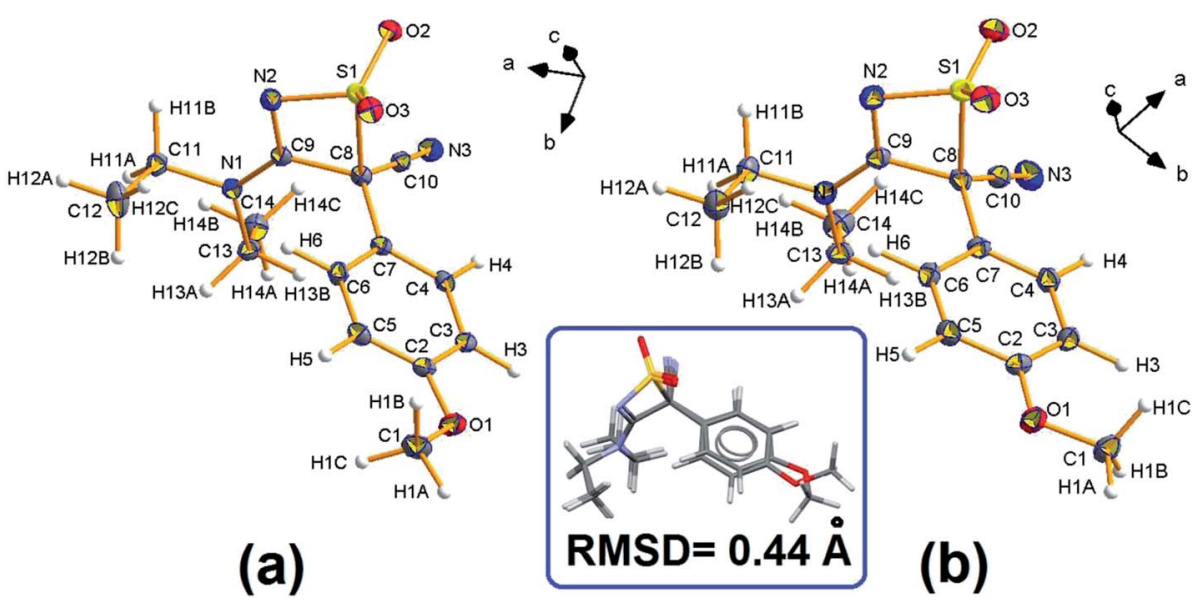

Fig. 1 (a) Molecular structure of the asymmetric unit of DTC in the crystal form A, with the atom-numbering scheme. (b) Same as (a), in the crystal form B. Thermal ellipsoids, drawn at the $50 \%$ probability level, refer to the structures at $T=100 \mathrm{~K}$. Inset: best least-squares superposition of the two asymmetric units, overlaid onto the thiazete group, with the corresponding root mean square deviation.

mass distances, $d_{\mathrm{CM}}$, within the whole packing sphere with $d_{\mathrm{CM}}$ $\leq 13 \AA$ A reduces by $1.11(6) \%$ in form A and $1.21(8) \%$ in form B. Lowering $T$ implies thus just a rescaling of the intermolecular centre of mass distances, without significantly affecting the main packing features of either crystal form.

\subsection{Crystal packing}

Fig. 2 compares the Hirshfeld surface fingerprint plots $^{47}$ for A and B at $T=100 \mathrm{~K}$. A full list of short intermolecular contacts is available in the ESI (Section S3, Tables S7-S10†). The general shape of the plots is similar, and actually, the relative amount of surface bins attributable to possibly favorable interactions is similar as well.

Fig. 3 compares the fingerprint plot coverage of different atom pairs, i.e. it represents the relative amount of the total
Hirshfeld surface that is covered by the $\left(d_{\mathrm{i}}+d_{\mathrm{e}}\right)$ pixels attributed to various $\mathrm{X} \cdots \mathrm{H}(\mathrm{X}=\mathrm{N}, \mathrm{O}, \mathrm{C}, \mathrm{H})$ distances. In both polymorphs, $\mathrm{H} \cdots \mathrm{H}$ contacts are the most frequent ones, as it usually occurs in hydrogen-rich crystals. The slight increment of $\mathrm{N}, \mathrm{O} \cdots \mathrm{H}$ frequencies in $\mathrm{B}$ occurs at the expenses of the $\mathrm{C} \cdots \mathrm{H}$ frequency, while other atom-atom close contacts are much less relevant.

Translating the relative picture on absolute grounds, we note that some features that are clearly visible in A become barely appreciable, or even disappear, in B (Fig. 2). The four spikes in $\mathrm{A}$, at a total distance $\left(d_{\mathrm{i}}+d_{\mathrm{e}}\right)$ of $\approx 2.4 \AA$ and $\approx 2.5 \AA$, are the signature of short $\mathrm{H} \cdots \mathrm{O}$ and $\mathrm{H} \cdots \mathrm{N}$ contacts, respectively. In $\mathrm{B}$, they shift at higher $d_{\mathrm{i}}+d_{\mathrm{e}}$ and are smoothed down into the $\mathrm{H} \cdots \mathrm{H}$ background in central region of the diagram. The two lateral wings in $\mathrm{A}$ at $d_{\mathrm{i}}+d_{\mathrm{e}} \approx 2.7 \AA$, which are due to close $\mathrm{C} \cdots \mathrm{H}$ contacts and are partly attributable to $\mathrm{C}-\mathrm{H} \cdots \pi$ interactions, somewhat survive in B at longer distances, but again they are
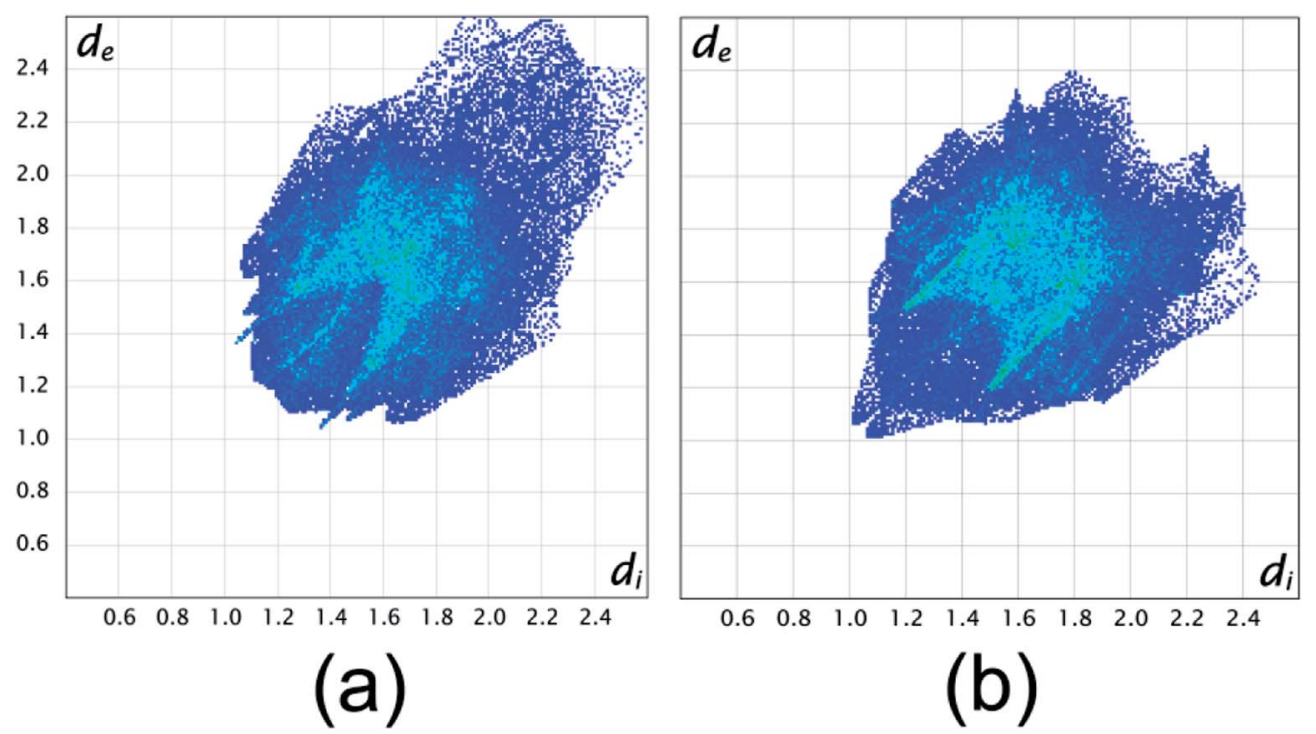

Fig. 2 (a) Hirshfeld surface fingerprint plot for the asymmetric unit of form $A$ at $T=100 \mathrm{~K}$. The plot shows the frequency of intermolecular pairs of internal $\left(d_{i}\right)$ and external $\left(d_{\mathrm{e}}\right)$ distances describing the closest atomic contacts with the in-crystal molecular Hirshfeld surface. Light blue points mean increasing frequency. (b) Same as (a), referring to the asymmetric unit of B. 


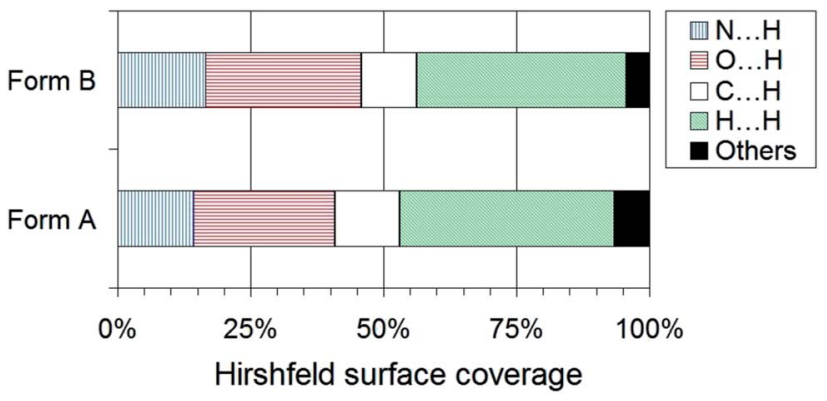

Fig. 3 Relative contributions to Hirshfeld surface area for the close molecular contacts in DTC forms A and B.

not neatly distinguishable anymore. In general, close hydrogenacceptor contacts roughly conserve their relative occurring frequencies in both the crystal structures, even though $\mathrm{O} \cdots \mathrm{H}$ and $\mathrm{N} \cdots \mathrm{H}$ interactions are slightly more frequent in the polymorph B (Fig. 3). Overall, though, these differences are barely significant, accounting for changes lower than $2.5 \%$ in the relative coverage of Hirshfeld surfaces, as expected for weak hydrogen bonded contacts in polymorphs of organic crystals. ${ }^{66}$ In general, both the crystal forms of DTC are characterized by similar packing modes, with the A form exploiting a slightly higher packing efficiency, as testified by a lower cell volume (Section 3.1) and by the fact that, on average, similar intermolecular contacts are translated at longer distances in the $\mathrm{B}$ phase. These evidences suggest that intermolecular NCI might be somehow weaker in this phase.

\subsection{Conformational interconversion paths}

In the gas phase, the two DTC conformers have essentially the same energy after full coordinate optimization. At the M06/pobTZVP theory level here adopted, and taking into account the vibrational zero-point corrections, the $\mathrm{B}$ conformer is more stable by just $8.64 \times 10^{-5} \mathrm{~h}\left(0.05 \mathrm{kcal} \mathrm{mol}^{-1}\right)$ than the A one. STQN calculations (Section 2.5) estimate that the rotation barrier of the terminal $-\mathrm{OCH}_{3}$ group which relates the two minima (Section 3.1) is $3.2 \mathrm{kcal} \mathrm{mol}^{-1}$ high, i.e. comparable with that associated to internal rotations in ethane (2.8$\left.2.9 \mathrm{kcal} \mathrm{mol}^{-1}\right) \cdot{ }^{67}$ Accordingly, it can be expected that free interconversion of the isolated molecule between the stable DTC conformers easily occurs at ordinary temperatures. DTC is thus an interesting test case for studying how different conformations, equally populated in the Boltzmann's sense before nucleation, give rise to crystal structures with different relative stability. This is probably due to the fact that the rotation of the methoxy group has a neat influence on the distribution and strength of intermolecular contacts (see below).

In the crystal, at variance with the gas-phase results, the conformer A becomes invariably more stable than the B one by, on average, $1.20(4) \mathrm{kcal} \mathrm{mol}^{-1}$. This is likely related to a slight more favorable conformation of the whole molecule, which implies small adjustments in the dihedral angles that describe the mutual orientation of the phenyl and thiazete rings and the relative position of the $-\mathrm{NEt}_{2}$ alkyl chains (Section 3.1). However, the rotation of the methoxy moiety must be hindered in the solid state, as spontaneous interconversion of the two polymorphs was never observed (see below).

\subsection{Virtual polymorph screening}

To gain insights into the relative interplay of molecular conformation and crystal packing, either conformer was fully optimized within the lattice of the other one,${ }^{19}$ comparing the virtual structures so generated with the actual ones. Table 1 shows the corresponding lattice energies, as obtained from full optimization (atomic coordinates and cell parameters) of the corresponding crystal structures (Section 2.4). No significant differences were detected in the crystal packing of the fully optimized A and B polymorphs with respect to the original X-ray structures. Moreover, in agreement with previous studies on virtual polymorphs, ${ }^{19,68,69}$ both the hypothetic structures formed by the A lattice allocating the $\mathrm{B}$ conformer $(\mathrm{A}[\mathrm{B}])$ and those with exchanged roles $(\mathrm{B}[\mathrm{A}])$ correspond to a minimum on the electronic potential energy surface. This means that the $P 2_{1} / n$ DTC lattice has enough flexibility to fit molecules of different shape. A change in the translational invariance conditions is sufficient to relax the unfavorable NCI (see below) and/or to strengthen the favorable ones. Even though it is still possible that a symmetry lowering (increase) to suitable subgroups (supergroups) will result in even more stable DTC polymorphs, no experimental evidence is available up to now of crystal forms different from $P 2_{1} / n$. A broader experimental polymorph screening might disclose further hints on this topic.

As for the present case, the optimized unit cells of the virtual structures (Table S11 ESI $\dagger$ ) are markedly different with respect to the real ones. Overall, the cell volumes of the A and B lattices change by $-0.6 \%$ and $+6.4 \%$ when they are forced to allocate the "wrong" conformers. In both cases, the $c$ cell edges undergoes a significant increase $(\mathrm{A}[\mathrm{B}]:+4.6 \% ; \mathrm{B}[\mathrm{A}]$ : $+3.8 \%)$, while the monoclinic angle sharpens $(\mathrm{A}[\mathrm{B}]$ : $-2.0 \% ; \mathrm{B}[\mathrm{A}]$ : $-4.9 \%$ ). In A, however, both the $a$ and $b$ edges become shorter by $\sim-2.6 \%$ (Table S11 ESI $\dagger$ ), overall resulting in a cell volume and density very similar to the original $\mathrm{A}[\mathrm{A}]$ one (Table 1 ). However, lattice energies of the virtual $\mathrm{A}[\mathrm{B}]$ and $\mathrm{B}[\mathrm{A}]$ structures are invariably less favorable than the real $\mathrm{A}[\mathrm{A}]$ and $\mathrm{B}[\mathrm{B}]$ ones

Table 1 Matrix of the lattice energies per molecule $\left(\mathrm{kcal} \mathrm{mol}^{-1}\right)$ and densities $\left(\mathrm{g} \mathrm{cm}^{-3}\right)$ of real (bold) and virtual ${ }^{a}$ polymorphs of $\mathrm{DTC}^{b}$

\begin{tabular}{|c|c|c|c|c|}
\hline \multirow{2}{*}{$\frac{\text { Conformer } \rightarrow}{\text { Structure } \downarrow}$} & \multicolumn{2}{|c|}{ Energies } & \multicolumn{2}{|c|}{ Densities } \\
\hline & A & B & A & B \\
\hline A & -43.0 & -39.1 & 1.502 & 1.510 \\
\hline B & -34.4 & -42.7 & 1.441 & 1.533 \\
\hline
\end{tabular}

${ }^{a}$ Virtual structures were generated starting from the real ones, upon a $\sim 170$ deg wide rotation of the terminal $-\mathrm{OCH}_{3}$ group in the asymmetric unit. ${ }^{b}$ All the crystal structures were fully optimized at $0 \mathrm{~K}$ with the CRYSTAL14 program by relaxing at the same time cell parameters and atomic coordinates at the same M06/pob-TZVP theory level employed in single-point calculations. Standard convergence criteria $^{37}$ on energy and its gradient were employed. Lattice energies take into account the conformational relaxation of the asymmetric unit and are expressed in kcal per mol per molecule. 
(Table 1). Interestingly, the B structure is the less suited to allocate the other conformer, as lattice adjustments would require up to $\sim+8 \mathrm{kcal}$ per mol per molecule, while the A one can fit the $\mathrm{B}$ conformer with a $\sim+4 \mathrm{kcal}$ per mol expense per molecule.

This procedure suggests that there is a $1: 1$ correspondence among the observed structures and the observed molecular conformations. A hypothetical conformational change in the solid state is never fully compatible with the pristine crystal lattice. This complies well with the existence of a high kinetic barrier against the interconversion of the two polymorphs in the solid state, which indeed was never observed.

\subsection{Lattice cohesive energies and energy ranking}

Fig. 4 shows the total M06 cohesive energies (Section 2.6) as a function of $T$, each computed from the corresponding (TLS + $\mathrm{H}$ )-corrected (Section 2.3) experimental structure. Table S12 $\mathrm{ESI} \dagger$ shows the corresponding numerical entries. In both polymorphs, the lattice energy mirrors the quadratic behavior of the

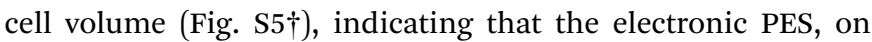
average, is well described by a harmonic potential. Interestingly, the distance between the $E_{\text {coh }}$ 's curves linearly decreases with $T$, and becomes $\sim 0.4 \mathrm{kcal} \mathrm{mol}^{-1}$ per molecule smaller at $100 \mathrm{~K}$. However, assuming that the quadratic form of the potential holds true also for $T<100 \mathrm{~K}$, the two curves should never cross down to $0 \mathrm{~K}$, where the estimated cohesive energies per molecule are $E_{\text {coh }}(\mathrm{A})=-19.6(2) \mathrm{kcal} \mathrm{mol}^{-1}$ and $E_{\text {coh }}(\mathrm{B})=$ $-17.8(3) \mathrm{kcal} \mathrm{mol}^{-1}$.

On the basis of M06 calculations, DTC is likely monotropic and $\mathrm{B}$ is the metastable phase, in agreement with the predictions at $T=0 \mathrm{~K}$ on the fully optimized structures (Section 3.5). Moreover, the following experimental, crystallographic and electronic evidences substantiate the M06 outcomes. (i) The molecular conformer present in the A form is slightly more

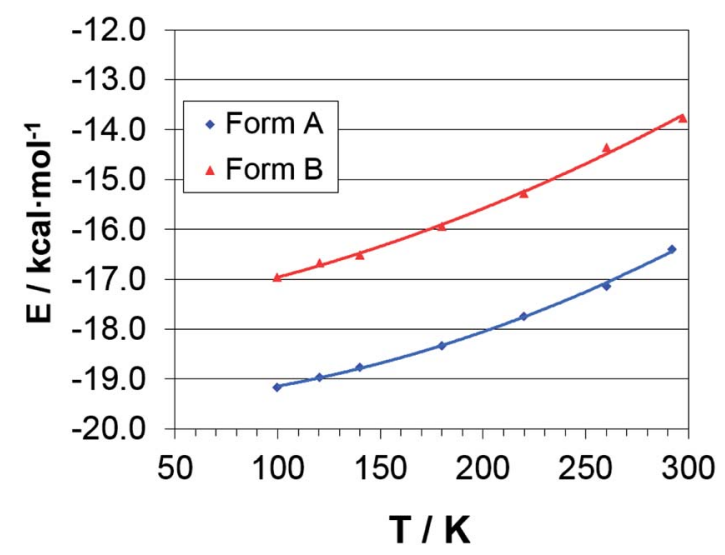

Fig. 4 Lattice cohesive energies, $E_{\text {coh, }}$ of the $A$ (blue rhombi) and $B$ (red triangles) DTC polymorphs per molecule, as evaluated by periodic calculations at the M06 pob-TZVP theory level, from the experimental structures as a function of $T$. The corresponding linear least-squares fits have equations $E_{\text {coh }}=2.8(6) \times 10^{-5} \times T^{2}+5(4) \times 10^{-3} \times T-$ 17.8(1) with $R^{2}=0.9965$ for the form $\mathrm{B}$ (red line) and $E_{\mathrm{coh}}=3.4(5) \times$ $10^{-5} \times T^{2}+1(2) \times 10^{-3} \times T-19.6(2)$ with $R^{2}=0.9987$ for the form $A$ (blue line). stable than that present in the B form (Section 3.1); (ii) in the form A, molecules are packed more closely, setting up somewhat shorter favorable interatomic contacts than in the B one (Section 3.3). (iii) The in-crystal molecular dipole moment of the polymorph A is larger, and thus stronger attractive electrostatic interactions are set up (Section 3.8 below). We thus believe that the present M06/pob-TZVP correctly reproduce the relative stability of the two crystal forms, at least on a common, relative energy scale. It should be stressed, however, that absolute energy differences are quite low (less than 2 kcal per mol per molecule) and their magnitudes depend on the theoretical framework. For example, dispersion-corrected PBE0+D/pobTZVP simulations even result in positive $E_{\text {coh }}$ estimates (Table S13 ESI $\dagger$ ), even though the system remains monotropic.

It can be thus concluded that the mutual conversion between the two forms is thermodynamically not possible; in principle, only B could spontaneously transform into A, but such a phase change was never observed since the synthesis of B in $2010 .^{22}$ This evidence can be explained with (i) the high crystal-field induced kinetic barrier that hampers free rotation of the $-\mathrm{OCH}_{3}$ group in the DTC asymmetric unit, and (ii) the significant lattice rearrangement to which the $\mathrm{B}$ structure would undergo to reallocate the A-compatible conformer (Section 3.5).

\subsection{Energy decomposition}

To gain insights into the relative weight of different NCI in determining the cohesive energy of the two crystal forms, it is desirable to decompose $E_{\text {coh }}$ into terms that can be associated with specific physical-chemical classes of intermolecular interactions. ${ }^{18}$ The interested reader can find a full discussion on the comparison of different computational recipes in the ESI (Section S4 ESI $\dagger$ ). We here refer to the outcomes from Spackman's ECDA model on the M06/pob-TZVP charge densities, as this method quantitatively reproduces the trend of moleculemolecule interaction energies $\left(E_{\text {int }}\right) v s$. their centre-of-mass distances, as estimated from the corresponding full quantum calculations. In any case (Section S4 ESI), all the computational recipes we tested agree very well in their general trends, and even quantitatively as concerns the electrostatic terms (Fig. S7 ESI $\dagger$ ). Therefore, the general conclusions remain the same irrespective of the specific energy decomposition scheme adopted.

Fig. 5 shows the ECDA trends of electrostatic and dispersiverepulsive contributions to total cohesive energies for the A and B phases as a function of $T$. The corresponding tabular entries are provided in Table S14 ESI. $\dagger$ Electrostatic energies dominate in both forms, but their relative weight with respect to $\left(E_{\mathrm{dis}}+\right.$ $\left.E_{\text {rep }}\right)$ becomes smaller at higher $T$. In fact, $\left(E_{\text {el }}\right)$ and $\left(E_{\text {dis }}+E_{\text {rep }}\right)$ have always opposite slopes as a function of $T$, with electrostatic terms becoming more stabilizing upon cooling (Fig. 5a) and van der Waals ones upon warming. A close look to individual energy contributions (Fig. 5b, Table S14 ESI $\dagger$ ) shows that, upon cooling, the overall $\left|E_{\text {rep }} / E_{\text {dis }}\right|$ ratio linearly increases in both polymorphs. As molecules come closer and closer while the cell volume shrinks (Section 3.1, Fig. S5 ESI $\dagger$ ), repulsions increase their relative importance over dispersions. However, lowering $T$ 


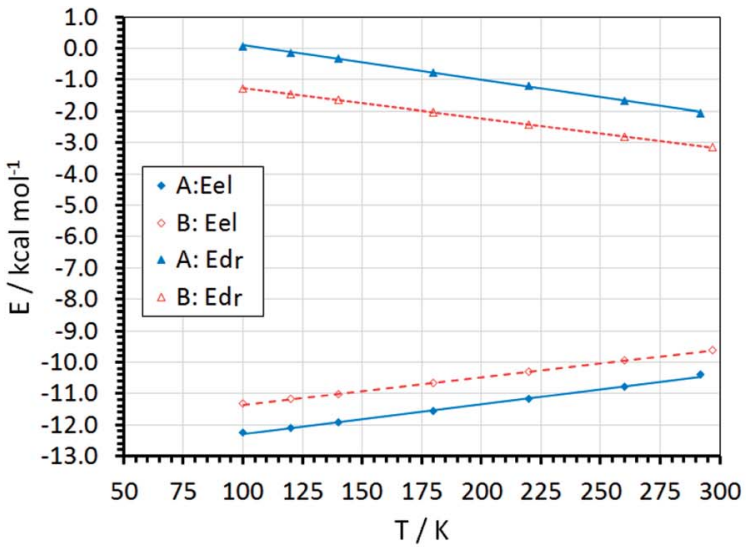

(a)

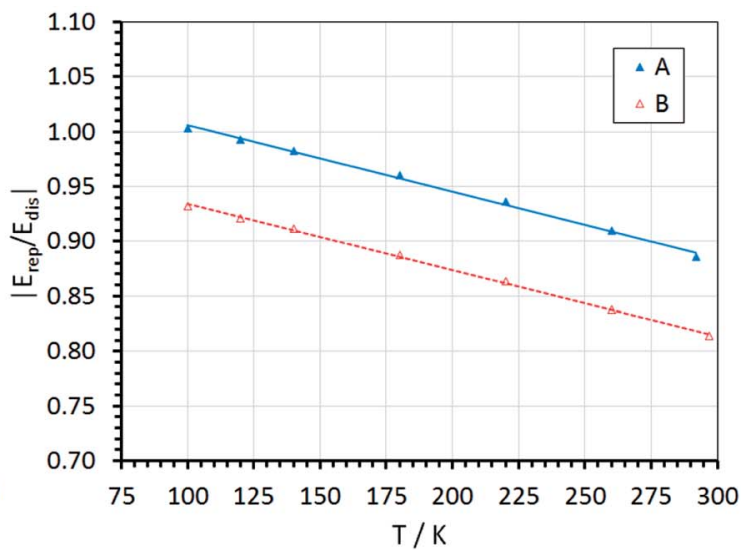

(b)

Fig. 5 Energy contributions, in $\mathrm{kcal} \mathrm{mol}^{-1}$, to the total cohesive energies, $E_{\mathrm{coh}}$, of the $\mathrm{A}$ (full blue points) and $\mathrm{B}$ (open red points) lattices as a function of $T$. Diamonds: electrostatic energy $\left(E_{\mathrm{el}}\right)$; triangles: dispersive-repulsive energy $\left(E_{d r}=E_{\text {dis }}+E_{\text {rep }}\right)$. (a) ECDA energies. (b) $\left|E_{\text {rep }} / E_{\text {dis }}\right|$ ratio.

implies rigid translations of molecular units toward each other (Section 3.2), i.e. no significant conformational changes take place to relieve unfavorable atom-atom contacts. This means that cohesion in DTC is not driven by pure dispersive-repulsive balance; otherwise, symmetry-dependent molecules would rearrange themselves to adapt to the mutated thermodynamic boundary conditions by maximizing $E_{\text {dis }}$ and/or reducing $E_{\text {rep }}$. Yet, neither structural discontinuities nor significant conformational changes were appreciable in either phase, in strict agreement with the ECDA predictions. Thus, we conclude that the present study demonstrates that electrostatic energy terms are invariably the dominant ones (Fig. 5) and mostly determine the crystal field.

\subsection{In-crystal dipole moments}

To gain a deeper understanding of DTC electrostatics, in-crystal molecular dipole moments, $\mu$, were evaluated from the M06/ pob-TZVP periodic densities by exploiting the AIM theory (Section 2.4). ${ }^{70}$ In this framework, each dipole can be decomposed into an atomic polarization component, $\mu_{\mathrm{P}}$, given by the vector sum of atomic first moments $\left(\boldsymbol{\mu}_{\mathrm{P}}=\sum_{\Omega} \boldsymbol{\mu}(\Omega)\right)$, plus a charge transfer (CT) term, $\mu_{\mathrm{CT}}=\left[\sum_{\Omega} q(\Omega) \cdot \mathbf{r}_{\Omega}\right], q(\Omega)$ being the net atomic charge associated to the $\Omega$ atomic basin, and $\mathbf{r}_{\Omega}$ the corresponding nuclear coordinate vector. Being DTC neutral, both $\mu_{\mathrm{P}}$ and $\mu_{\mathrm{CT}}$ are origin-independent and retain a physical meaning in their own. ${ }^{23,70}$ Parameters used to assess the quality of the integration of the charge density, such as the residual charge of the unit cell and the absolute values of atomic Lagrangian integrals, were all satisfactorily low, being on average as low as $0.0115(8)(0.0108(7)) e$ and $4.6(4) \times 10^{-4}(5.5(3)$

$\left.\times 10^{-4}\right) e \AA^{-2}$, respectively, for the form A(B). Table 2 reports the modules of the various polarization and charge transfer vectors, as well as the angle between them $(\gamma)$, for the asymmetric unit of both polymorphs as a function of $T$. As it is often the case, the two contributions oppose each other as atoms customarily polarize in a direction counter to the local electric field caused by the interatomic charge transfer; indeed, $\gamma$ was found to be close to $180 \mathrm{deg}$ within $10-25 \mathrm{deg}$.

As expected on the basis of the more favorable electrostatic energies (Section 3.7, Fig. 5), the DTC molecule bears a $\sim 0.8-$ $0.9 D$ larger dipole module in the form A. Moreover, in both polymorphs the dipole magnitude increases at low T. Fig. 6 shows the relative changes of $\left|\mu_{\mathrm{P}}\right|$ and $\left|\mu_{\mathrm{CT}}\right|$ modules with respect to the room temperature structures. The dominant charge transfer contributions (Table 2) are essentially independent on $T$, as they just exhibit very small $(<1 \%)$ adjustments down to $100 \mathrm{~K}$. More significant are the relative changes of the $\left|\mu_{\mathrm{P}}\right|$ terms, whose modules show a neat tendency to undergo up to a $\sim 4 \%$ reduction upon decreasing $T$. Since $\mu_{\mathrm{P}}$ is opposed to $\mu_{\mathrm{CT}}$, the total dipole moments increase at lower $T$. Recently, some of us demonstrated ${ }^{23}$ that the crystal field has a significant enhancing effect on the dipole moment of the isolated DTC molecule. In particular, crystal packing forces the atomic polarization to decrease and makes it less effective in counteracting CT contributions, in agreement also with previous studies. ${ }^{71,72}$ Therefore, a decrease of $\left|\mu_{\mathrm{P}}\right|$ might be considered as

Table 2 In-crystal M06/pob-TZVP AIM atomic polarization $\left(\mu_{P}\right)$ and charge transfer $\left(\mu_{\mathrm{CT}}\right)$ vector module contributions to the total dipole vector $(\boldsymbol{\mu})$ of $A$ and $B$ forms of DTC, as a function of $T$. The angle between the $\boldsymbol{\mu}_{\mathrm{P}}$ and $\boldsymbol{\mu}_{\mathrm{CT}}$ vectors is also given. Measure units are Kelvin, Debye and degrees

\begin{tabular}{|c|c|c|c|c|c|c|c|c|c|}
\hline \multicolumn{5}{|c|}{ Form A } & \multicolumn{5}{|c|}{ Form B } \\
\hline$T$ & $\left|\mu_{P}\right|$ & $\left|\mu_{\mathrm{CT}}\right|$ & $|\mu|$ & $\gamma$ & $T$ & $\left|\mu_{P}\right|$ & $\left|\mu_{\mathrm{CT}}\right|$ & $|\mu|$ & $\gamma$ \\
\hline 100 & 4.39 & 16.52 & 12.23 & 169.9 & 100 & 3.13 & 14.11 & 11.30 & 156.9 \\
\hline 120 & 4.39 & 16.53 & 12.23 & 169.9 & 120 & 3.15 & 14.12 & 11.31 & 156.6 \\
\hline 140 & 4.44 & 16.50 & 12.16 & 170.0 & 140 & 3.17 & 14.14 & 11.30 & 156.6 \\
\hline 180 & 4.43 & 16.48 & 12.14 & 169.9 & 180 & 3.20 & 14.14 & 11.26 & 156.9 \\
\hline 220 & 4.47 & 16.46 & 12.09 & 170.0 & 220 & 3.24 & 14.17 & 11.26 & 156.9 \\
\hline 260 & 4.49 & 16.43 & 12.04 & 170.0 & 260 & 3.27 & 14.19 & 11.24 & 157.3 \\
\hline 292 & 4.55 & 16.42 & 11.97 & 169.4 & 297 & 3.27 & 14.15 & 11.23 & 156.5 \\
\hline
\end{tabular}




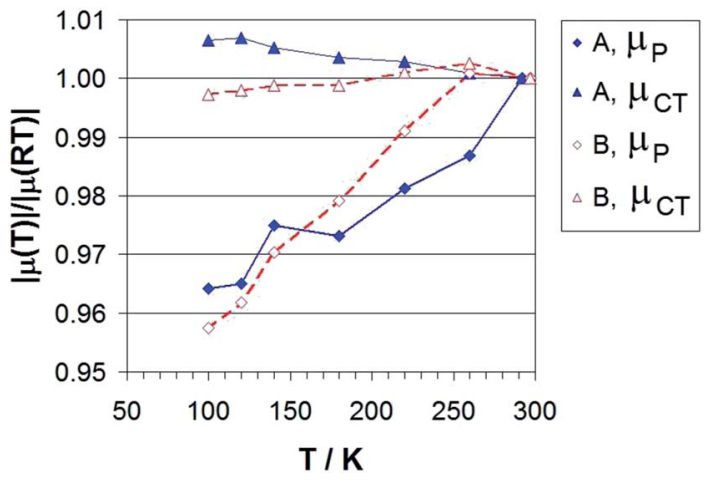

Fig. 6 Behavior of the polarization, $\left|\mu_{P}\right|$ (diamonds), and charge transfer, $\left|\boldsymbol{\mu}_{\mathrm{CT}}\right|$ (triangles), contributions to the total in-crystal dipole vector module of the DTC molecule in forms A (full blue points) and B (empty red points), as a function of $T$. All quantities derive from the integration of the corresponding periodic M06/pob-TZVP charge densities, according with the AIM theory. Values normalized to RT estimates are provided. The lines are guides for the eye.

a sign of an increased strength of the crystal field. This complies well with the increasing importance of electrostatic contributions to the total lattice energy at low $T$. As molecules become closer and closer, their local electric fields cooperate more effectively, determining a shift of the system toward more attractive regions of the Coulomb and polarization potentials.

\section{Conclusions}

In this work, the two known conformational polymorphs of DTC, a thermally stable azidation by-product of some isothiazole derivatives that exhibits a significant similarity with $\beta$ sultamic drugs, ${ }^{21}$ were studied among RT and $100 \mathrm{~K}$. Single crystal X-ray diffraction experiments were complemented by accurate quantum mechanical and semiempirical simulations, both in the solid state and in the gas phase, to disclose how energetic and structural factors cooperate in giving rise to different crystal packings. In this respect, DTC is an ideal test case to investigate the interplay of dispersive and electrostatic interactions, as it lacks strong hydrogen bonds and does not undergo structural phase transitions in the $T$ range here explored.

The two DTC forms, A and B, are very similar from a general crystallographic viewpoint. They both have the same $P 2_{1} / n$ symmetry and comparable cell volumes, densities and thermal expansion coefficients. The most striking difference is due to the relative orientation of a terminal $-\mathrm{OCH}_{3}$ group, which in the phase B is rotated by $\sim 170 \mathrm{deg}$ with respect to the asymmetric unit in the phase A. Lowering $T$ implies just a reduction of the intermolecular centre of mass distances, and does not significantly affect conformations and mutual orientations of neighboring molecules. Accordingly, most intermolecular close contacts are similar in the two polymorphs.

In the gas phase, the rotation barrier of the terminal $-\mathrm{OCH}_{3}$ group in DTC is as low as $3.2 \mathrm{kcal} \mathrm{mol}^{-1}$. This rotation is kinetically hindered in the solid state, as the system is monotropic in the $100 \mathrm{~K}-\mathrm{RT}$ range, with the phase B as the metastable one. Virtual polymorph screening suggests that there is a $1: 1$ correspondence among the observed lattice structures and the solid-state molecular conformations. A hypothetical conformational change in the asymmetric unit is never compatible with the pristine crystal lattice, as it produces unfavorable intermolecular interactions that must be relieved at the expense of the lattice cohesive energy.

In both polymorphs, the $T$-driven reduction of the cell volume increases the strength of electrostatic interactions and, in particular, decreases the atomic dipolar polarization terms and cause the total molecular dipole moment to increase. At the same time, dispersive interactions become less effective, as repulsive terms are enhanced by closer proximity of neighbouring molecules at lower temperatures. These results do not depend on the specific computational method employed to decompose the crystal cohesive energy into individual NCI terms. This conformity of views provides in our opinion solid grounds to our interpretation of crystal field effects of DTC as dominated by electrostatics, and in particular by permanent molecular dipole moments.

It should be remarked that the title compound was selected as a practical test case where the crystal packing is clearly governed by the balance among dispersive/repulsive and electrostatic factors. The presence of strong hydrogen bond patterns, as well as more than one molecule in the asymmetric unit, would likely complicate the picture. The occurring of strong hydrogen bond patterns will likely cooperate with electrostatics, relegating the dispersive/repulsive balance to a less central role. Structural and energetic investigations as a function of $T$ on other suitable test cases will provide further insights on this topic.

In conclusion, we showed that multi- $T$ single-crystal X-ray diffraction experiments, combined with quantum mechanical and semiempirical calculations, allow to go a step further with respect to the static view provided by usual computational polymorph screening, which focuses on the most stable structures at $0 \mathrm{~K}$ and does not explicitly take into account finite- $T$ effects. The relative weights of electrostatic and dispersive/ repulsive interactions were investigated as a function of $T$, and a tentative explanation for the reasons behind monotropism in DTC was proposed. In addition, the present study provides accurate experimental molecular geometries and thermal expansion coefficient estimates in a couple of subtly different polymorphs, and might be useful as a reference test bench for predictive calculations of thermal properties and crystal structures.

\section{Conflicts of interest}

There are no conflicts of interest to declare.

\section{Acknowledgements}

The Authors thank prof. F. Clerici for provision of the title compound. Thanks are also due to prof. A. Gavezzotti for fruitful discussions. The LISA program sponsored by CINECA and Regione Lombardia is gratefully acknowledged for 
provision of computing time (project MODEFRUC) and the Danish National Research Foundation is acknowledged for partial funding of this work through contract grant number DNRF93. This research was partially funded by the Unimi Development Plan-Line 2, Action B, project NOVAQ, no. PSR2015-1716FDEMA_08. Mr P. Colombo of the Italian CNR is also to be warmly thanked for technical assistance.

\section{References}

1 A. O. F. Jones, B. Chattopadhyay, Y. H. Geerts and R. Resel, Adv. Funct. Mater., 2016, 26, 22335.

2 Design of Organic Solids, in Top. Curr. Chem., ed. E. Weber, Springer-Verlag: Berlin-Heidelberg, 1998, vol. 198.

3 A. J. Cruz-Cabeza and J. Bernstein, Chem. Rev., 2014, 114, 2170.

4 R. G. Desiraju, Nat. Mater., 2002, 1, 77.

5 T. Threlfall, Org. Process Res. Dev., 2003, 7, 1017.

6 S. L. Price, Acta Crystallogr., Sect. B: Struct. Sci., Cryst. Eng. Mater., 2013, 69, 313.

7 A. Gavezzotti and G. Filippini, Chem. Commun., 1998, 287.

8 G. M. Day, A. V. Trask, W. D. S. Motherwell and W. Jones, Chem. Commun., 2006, 54-56.

9 W. C. McCrone, Polymorphism, Physics and Chemistry of the Organic Solid State, ed. D. Fox, M. M. Labes and A. Weissberger, Interscience Publishers, New York, 1965; vol. 2; p. 725.

10 A. M. Reilly, R. I. Cooper, C. S. Adjiman, S. Bhattacharya, A. D. Boese, J. G. Brandenburg, P. J. Bygrave, R. Bylsma, J. E. Campbell, R. Car, et al., Acta Crystallogr., Sect. B: Struct. Sci., Cryst. Eng. Mater., 2016, 72, 439.

11 M. A. Neumann, F. J. J. Leusen and J. Kendrick, Angew. Chem., Int. Ed., 2008, 47, 2427.

12 A. Gavezzotti, Acc. Chem. Res., 1994, 27, 309.

13 G. R. Desiraju, Angew. Chem., Int. Ed., 2007, 46, 8342.

14 S. L. Price and S. M. Reutzel-Edens, Drug Discovery Today, 2016, 6, 912.

15 A. Gavezzotti, Molecular Aggregation. Structural Analysis and Molecular Simulations of Crystals and Liquids, IUCr book series, Oxford University Press, Oxford, 2007.

16 L. Lo Presti, R. Soave and R. Destro, J. Phys. Chem. B, 2006, 110, 6405.

17 R. Destro, E. Sartirana, L. Loconte, R. Soave, P. Colombo, C. Destro and L. Lo Presti, Cryst. Growth Des., 2013, 13, 4571.

18 G. Macetti, L. Loconte, S. Rizzato, C. Gatti and L. Lo Presti, Cryst. Growth Des., 2016, 16, 6043.

19 M. Causà and R. Centore, CrystEngComm, 2017, 19, 1320.

20 M. Salvalaglio, C. Perego, F. Giberti, M. Mazzotti and M. Parrinello, Proc. Natl. Acad. Sci. U. S. A., 2015, 112, E6.

21 F. Clerici, M. L. Gelmi, R. Soave and L. Lo Presti, Tetrahedron, 2002, 58, 5173.

22 A. M. Orlando, L. Lo Presti and R. Soave, Acta Crystallogr., Sect. E: Struct. Rep. Online, 2010, 66, o2032.

23 L. Lo Presti, A. M. Orlando, L. Loconte, R. Destro, E. Ortoleva, R. Soave and C. Gatti, Cryst. Growth Des., 2014, 14, 4418.

24 Bruker, SMART and SAINT, Bruker AXS Inc., 1999, Madison, Wisconsin, USA.
25 G. M. Sheldrick, SADABS: Program for scaling and corrections of area-detector data, University of Göttingen, Germany, 1996.

26 G. M. Sheldrick, Acta Crystallogr., Sect. A: Found. Crystallogr., 2008, 64, 112.

27 B. T. M. Willis and A. W. Pryor, Thermal Vibrations in Crystallography, Cambridge University Press, Cambridge, 1975.

28 V. Schomaker and K. N. Trueblood, Acta Crystallogr., Sect. B: Struct. Sci., 1998, 54, 507.

29 J. D. Dunitz, E. F. Maverick and K. N. Trueblood, Angew. Chem., Int. Ed. Engl., 1988, 27, 880.

30 F. H. Allen and I. J. Bruno, Acta Crystallogr., Sect. B: Struct. Sci., Cryst. Eng. Mater., 2010, 66, 380.

31 C. F. Macrae, I. J. Bruno, J. A. Chisholm, P. R. Edgington, P. McCabe, E. Pidcock, L. Rodriguez-Monge, R. Taylor, J. van de Streek and P. A. Wood, J. Appl. Crystallogr., 2008, 41, 466-470.

32 N. Wahlberg, P. Ciochón, V. Petriçek and A. Ø. Madsen, Cryst. Growth Des., 2014, 14, 381.

33 J. G. Brandenburg and S. Grimme, J. Phys. Chem. Lett., 2014, $5,1785$.

34 J. J. Holstein, P. Luger, R. Kalinowski, S. Mebs, C. Paulman and B. Dittrich, Acta Crystallogr., Sect. B: Struct. Sci., Cryst. Eng. Mater., 2010, 66, 568.

35 E. A. Zhurova, C. F. Matta, N. Wu, V. V. Zhurov and A. A. Pinkerton, J. Am. Chem. Soc., 2006, 128, 8849.

36 D. E. Hibbs, J. R. Hanrahan, M. B. Hursthouse, D. W. Knight, J. Overgaard, P. Turner, R. O. Piltz and M. P. Waller, Org. Biomol. Chem., 2003, 1, 1034.

37 R. Dovesi, V. R. Saunders, C. Roetti, R. Orlando, C. M. Zicovich-Wilson, F. Pascale, B. Civalleri, K. Doll, N. M. Harrison, I. J. Bush, P. D'Arco, M. Llunell, M. Causà and Y. Noël, CRYSTAL14 User's Manual, University of Torino, Torino, 2014.

38 Y. Zhao and D. G. Truhlar, Theor. Chem. Acc., 2008, 120, 215. 39 M. F. Peintinger, D. V. Oliveira and T. Bredow, J. Comput. Chem., 2013, 34, 451.

40 J. M. Skelton, E. Lora Da Silva, R. Crespo-Otero, L. E. Hatcher, P. R. Raithby and S. C. ParkerWalsh, Faraday Discuss., 2015, 177, 181.

41 M. Walker, A. J. A. Harvey, A. Sen and C. E. H. Dessent, J. Phys. Chem. A, 2013, 117, 12590.

42 N. Marom, A. Tkatchenko, M. Rossi, V. V. Gobre, O. Hod, M. Scheffler and L. Kronik, J. Chem. Theory Comput., 2011, 7, 3944.

43 R. F. W. Bader, Atoms in Molecules: A Quantum Theory, Clarendon Press, Oxford, 1990.

44 C. Gatti and S. Casassa, TOPOND14 User's Manual, 2014.

45 T. Bredow, P. Heitjans and M. Wilkening, Phys. Rev. B, 2004, 70, 115111.

46 M. A. Spackman and A. S. Mitchell, Phys. Chem. Chem. Phys., 2001, 3, 1518.

47 M. A. Spackman and D. Jayatilaka, CrystEngComm, 2009, 11, 19.

48 M. J. Turner, J. J. McKinnon, S. K. Wolff, D. J. Grimwood, P. R. Spackman, D. Jayatilaka and M. A. Spackman, 
CrystalExplorer17, University of Western Australia, 2017, http://hirshfeldsurface.net.

49 M. J. Frisch, G. W. Trucks, H. B. Schlegel, G. E. Scuseria, M. A. Robb, J. R. Cheeseman, G. Scalmani, V. Barone, et al., Gaussian 09 (Revision A.1), Gaussian, Inc., Wallingford CT, 2009.

50 C. Peng and H. B. Schlegel, Isr. J. Chem., 1993, 33, 449.

51 B. Civalleri, K. Doll and C. M. Zicovich-Wilson, J. Phys. Chem. B, 2007, 111, 26.

52 L. Lo Presti, R. Soave, M. Longhi and E. Ortoleva, Acta Crystallogr., Sect. B: Struct. Sci., Cryst. Eng. Mater., 2010, 66, 527.

53 S. F. Boys and F. Bernardi, Mol. Phys., 1970, 19, 553.

54 M. A. Spackman, Chem. Phys. Lett., 2006, 418, 158.

55 M. A. Spackman, Keynote Lecture KN23.28, Electrostatic and related properties from accurate charge density analyses, IUCR2005, Book of Abstracts, Firenze, 2005, vol. C5.

56 A. D. Buckingham, Physical Chemistry: An Advanced Treatise, ed. D. Henderson, Academic Press, New York, 1970, p. 349.

57 M. Barzaghi, PAMoC (Version 2010-07-27), Online User's Manual; CNR-ISTM, Institute of Molecular Science and Technologies, Milano, Italy, http://www.istm.cnr.it/ barz/ pamoc/.

58 A. L. Spek, Acta Crystallogr., Sect. D: Struct. Biol., 2009, 65, 148.

59 D. Cremer and J. A. Pople, J. Am. Chem. Soc., 1975, 97, 1354.
60 S. Bhattacharya, V. G. Saraswatula and B. K. Saha, Cryst. Growth Des., 2016, 16(1), 277.

61 V. G. Saraswatula and B. K. Saha, Cryst. Growth Des., 2015, 15, 593.

62 S. Bhattacharya and B. K. Saha, Cryst. Growth Des., 2013, 13, 3299.

63 B. K. Saha, S. A. Rather and A. Saha, Eur. J. Inorg. Chem., 2017, 28, 3390.

64 V. G. Saraswatula, S. Bhattacharya and B. K. Saha, New J. Chem., 2015, 39, 3345.

65 S. Bhattacharya and B. K. Saha, CrystEngComm, 2014, 16, 2340.

66 L. Lo Presti, CrystEngComm, 2018, 20, 5976-5989.

67 G. Ercolani, J. Chem. Educ., 2005, 82, 1703.

68 R. Centore, S. Fusco, F. Capone and M. Causà, Cryst. Growth Des., 2016, 16, 2260.

69 R. Centore, M. Causà, F. Cerciello, F. Capone and S. Fusco, CrystEngComm, 2014, 16, 9168.

70 R. F. W. Bader, A. Larouche, C. Gatti, M. T. Carroll, P. J. MacDougall and K. B. J. Wiberg, Chem. Phys., 1987, 87, 1142.

71 E. May, R. Destro and C. Gatti, J. Am. Chem. Soc., 2001, 123, 12248.

72 C. Gatti, B. Silvi and F. Colonna, Chem. Phys. Lett., 1995, 247, 135. 